\title{
RELATION BETWEEN GROUPS WITH BASIS PROPERTY AND GROUPS WITH EXCHANGE PROPERTY
}

\author{
A.Al Khalaf, M. Alkadhi
}

\begin{abstract}
A group $G$ is called a group with basis property if there exists a basis (minimal generating set) for every subgroup $H$ of $G$ and every two bases are equivalent. A group $G$ is called a group with exchange property, if $x \notin\langle X\rangle \wedge x \in\langle X \cup\{y\}\rangle$, then $y \in\langle X \cup\{x\}\rangle$, for all $x, y \in G$ and for every subset $X \subseteq G$.

In this research, we proved the following: Every polycyclic group satisfies the basis property. Every element in a group with the exchange property has a prime order. Every $p$-group satisfies the exchange property if and only if it is an elementary abelian $p$-group. Finally, we found necessary and sufficient condition for every group to satisfy the exchange property, based on a group with the basis property.
\end{abstract}

\section{Introduction}

A generating set $X$ is said to be minimal if it has no proper subset which forms a generating set. The subset $X$ of a group $G$ is called independent, if for all $x \in X, x \notin\langle X \backslash\{x\}\rangle$. Independent set $X$ is called a basis subgroup $\langle X\rangle$. In 1978 Jones [5] introduced an initial study of semigroups with the basis property. Jones [5] states that if $G$ is an inverse semigroup and $U \leq V \leq G$ then a $U$-basis for $V$ is a subset $X$ of $V$ which is minimal such that $\langle U \cup X\rangle=$ G.

Key Words: Groups, Basis property, Exchange property.

2010 Mathematics Subject Classification: Primary 20D25; Secondary 20D10.

Received: 31.07.2014

Accepted: 20.02.2015 
So a minimal generating set for $V$ is a $\emptyset$-basis. A basis property of universal algebra $A$ means that every two minimal (with respect to inclusion) generating set ( basis) of an arbitrary subalgebra of $A$ have the same cardinality [1].

\section{Basis property}

Definition 2.1 A group $G$ is called a group with basis property if there exists a basis minimal (irreducible) generating sets (with respect to inclusion) for every subgroup $H$ of $G$ and every two bases are equivalent (i.e. they have the same cardinality) [1].

Notice that finitely generated vector spaces have the property that all minimal generating sets have the same cardinality. Jones [5] introduce another concept which is state for inverse semigroup.

Definition 2.2 An inverse semigroup $S$ has the strong basis property if for any inverse subsemigroup $V$ of $S$ and inverse subsemigroup $U$ of $V$ any two $U$-bases for $V$ have the same cardinality.

Let $(\mathbb{Z},+)$ be an additive abelian group, then we can write $\mathbb{Z}=\langle 1\rangle=\langle 2,3\rangle$ even though $2 \notin\langle 3\rangle$ and $3 \notin\langle 2\rangle$. Thus $\mathbb{Z}$ does not have the basis property. Hence free groups do not have the basis property. The first results on the basis property of groups was in [6]. The author proved that a group with basis property is periodic, all elements of such a group have prime power order, and solvable. Therefore by [1] every finite $p$-group has a basis property, and the homomorphic image of every finite group with basis property is again a group with basis property, but in case of infinite group we have the following:

Remark 2.3 Let $G=\sum_{i=1}^{\infty} \mathbb{Z}_{p^{i}}$ be a direct sum of a cyclic $p$-group $P$, then one of homomorphic image is a quasicyclic group $K=\mathbb{Z}_{p^{\infty}}$, which is not a group with basis property, but the group $G$ is a group with basis property.

Lemma 2.4 Let $G$ be a group in which every element has prime power order, let $x \in G$ such that $|x|=p^{c}$ and $y \in G$ such that $|y|=q^{b}, p \neq q$ are primes. Then $x y \neq y x$.

Proof. Suppose that $x y=y x$, then $x y$ is an element of order $p^{c} q^{b}$, hence $x y$ has a composite order in $G$. This is contradiction with basis property[1], so $x y \neq y x$.

Proposition 2.5 Let $G$ be a finite nilpotent group. Then $G$ is a group with basis property if and only if $G$ is a primary group.

Proof. Suppose that $G$ is a finite nilpotent group with basis property. From [11] every finite nilpotent group is decomposable in a direct product of Sylow 
subgroups. Then

$$
G=G_{1} \times G_{2} \times \cdots \times G_{m}
$$

such that $G_{i}$ is a $p_{i}$-group for some primes $p_{i}, p_{i} \neq p_{j}$ if $i \neq j$. If $m>1$, then in $G$ there exists two commute elements with a prime power order. Hence we have a contradiction with lemma(2-4). Thus $G$ is a primary group. $[5]$.

Conversely, if $G$ is a primary group, then $G$ is a group with basis property

A classification of group with the basis property was announced by Dickson and Jones in [5], but as far as we can see this has yet to be published. However a classification of finite groups with the basis property was given by Al Khalaf [1] exploiting Higman's result, this classification requires a technical condition on the $p$-group and he proved the following theorem:

Theorem 2.6 [1]. Let a finite group $G$ be a semidirect product of a $p$ group $P=F i t(G)$ (Fitting subgroup) of $G$ by a cyclic $q-$ group $\langle y\rangle$, of order $q^{b}$, where $p \neq q$ ( $p$ and $q$ are primes $), b \in \mathbb{N}$.Then the group $G$ has basis property if and only if for any element $y \in\langle y\rangle, y \neq e$ and for any invariant subgroup $H$ of $P$ the automorphism $\varphi_{u}$ must define an isotopic representation on every quotient Frattini subgroup of $H$.

In [4], the author used some common results from both group and module theory using Maschke, Clifford and Krull-Schmidt, to classify the group with basis property.

Finally Jones [7] studied basis property from the point of view exchange properties.

Theorem 2.7 [3] Let $G$ be a semidirect product of abelian $p$-group $P$ by a cyclic $q$-group $\langle y\rangle$, of order $q^{b}$, where $p \neq q$ ( $p$ and $q$ are primes), $b \in \mathbb{N}$, which is defined automorphism $\varphi$ of order $q^{b}$ and $P$ has an exponent $p^{c}, c \in \mathbb{N}$. Then the group $G$ has basis property if and only if there exists a polynomial $g(x) \in \mathbb{Z}[x]$ such that satisfy the following conditions:

1. The polynomial $f(x)=\bar{\theta}(g(x))$ is irreducible over the field $G F(p)$, $f(x) \mid x^{q^{b}}-1$ and $f(x) \nmid x^{q^{b-1}}-1$.

2. $g^{m}(\varphi)=0$.

In this research we study special group with the basis property. The concept of exchange property and continued results as shown in [7] and [8]. 
Theorem 2.8 Let $G$ be a finite polycyclic group such that $G$ has a presentation [9]:

$G=\left\langle x, y: x^{p^{c}}=y^{q^{b}}=1, y^{-1} x y=x^{r}\right\rangle$,

such that $p \neq q(p$ and $q$ are primes $) b, c, r \in \mathbb{Z}^{+},(p, r-1)=1$ and

$$
r^{q^{b}} \equiv 1\left(\bmod p^{c}\right), r \not \equiv 1(\bmod p), 0 \leq r \leq p^{c} .
$$

Then $G$ is a group with the basis property if and only if it satisfies the following conditions:

$$
\begin{gathered}
p \equiv 1\left(\bmod q^{b}\right), \\
r^{q^{b-1}} \not \equiv 1(\bmod p) .
\end{gathered}
$$

Proof. Suppose that $G$ is a group with the basis property. From $(2-1)$ we have that $G$ is a semidirect product of cyclic $p$-group $\langle x\rangle,|\langle x\rangle|=p^{c}$ by a cyclic $q$-group $|\langle y\rangle|=q^{b}$, where $p \neq q$ ( $p$ and $q$ are primes) $b, c \in \mathbb{Z}^{+}$. Then from [1] $G$ is a Frobenius group with kernel $\langle x\rangle$ and complement $\langle y\rangle$. Thus by [3] we see that $p \equiv 1\left(\bmod q^{b}\right)$. Thus $(2-3)$ holds.

Assume that

$$
r^{q^{b-1}} \equiv 1(\bmod p) \text {. }
$$

Then $r^{q^{b-1}}=1+m p$ for some $m \in \mathbb{Z}^{+}$.Considering the non trivial elements $x^{p^{c-1}}, y^{q^{b-1}}$ and using $(2-1)$ and $(2-5)$ then we have:

$$
\begin{gathered}
y^{-q^{b-1}} x^{p^{c-1}} y^{q^{b-1}}=\left(y^{-q^{b-1}} x y^{q^{b-1}}\right)^{p^{c-1}}=\left(y^{-q^{b-1}-1}\left(y^{-1} x y\right) y^{q^{b-1}-1}\right)^{p^{c-1}} \\
=\left(y^{-q^{b-1}-1} x^{r} y^{q^{b-1}-1}\right)^{p^{c-1}}=\cdots=\left(x^{r^{q^{b-1}}}\right)^{p^{c-1}}=x^{p^{c-1}(1+m p)}= \\
x^{p^{c-1}}\left(x^{p^{c}}\right)^{m}=x^{p^{c-1}} .
\end{gathered}
$$

Hence the $p$-element $x^{p^{c-1}}$ commutes with the $q$ - element in $G$, so we have a contradiction with lemma (2-4). Thus $(2-4)$ holds.

Conversely, let $G$ be a polycyclic group satisfying conditions $(2-3)$, and $(2-4)$. Then from [9] we see that $G$ is an extension of cyclic $p$-group $\langle x\rangle$ of order $p^{c}$ by cyclic $q$-group $\langle y\rangle$ of order $q^{b}, p \neq q$ ( $p$ and $q$ are primes) $b, c \in \mathbb{Z}^{+}$. Thus $(|\langle x\rangle|,|\langle y\rangle|)=1$ and $|G|=|\langle x\rangle||\langle y\rangle|$, then $\langle x\rangle \cap\langle y\rangle=\{1\}$ and $G=\langle x\rangle\langle y\rangle$, 
so $G=\langle x\rangle \rtimes\langle y\rangle$. Since $\langle x\rangle \unlhd G$ and $\langle x\rangle$ is an abelian $p$-group, then by using theorem (2-7) we prove that $G$ is a group with the basis property.

Now consider the polynomial $g(x)=x-r$ over the ring $\mathbb{Z}$. Denote that

$f(x)=\bar{\theta}(g(x))$. Then the polynomial $f(x)$ is an irreducible over the field $G F(p)$ and has $\bar{r}$ zeros. Thus by $(2-2)$, and $(2-4)$ we have $\bar{r}^{q^{b}}=1$, $\bar{r}^{q^{b-1}} \neq 1$, hence by Bezout theorem the polynomial $f(x)$ is divides $x^{q^{b}}-1$ and not divides $x^{q^{b-1}}-1$, i.e. the condition 1$)$ in theorem $(2-7)$ holds for $g(x)$. Now consider the automorphism $\varphi$, which defines a semidirect product $\langle x\rangle \rtimes\langle y\rangle$ and induced by $y$ element, i.e.

$$
\varphi: a \rightarrow y^{-1} a y, \quad \forall a \in\langle x\rangle .
$$

From $(2-1)$ we get

$$
\varphi(a)=a^{r}, \quad \forall a \in\langle x\rangle .
$$

Using additive form in $\langle x\rangle$, then we have $g(\varphi)=0$. Thus the codition 2) of theorem(2-7) for $g(x)$ holds too. Hence $G$ is a group with the basis property.

\section{$3 \quad$ Exchange property}

The fundamental property of generating operator $\varphi$ of subspace of the vector space $V$ over the field $F$ that this operator satisfies exchange property.

Definition 3.1 Let $V$ be a vector space, then $\forall x, y \in V$ and for every subset $X \subseteq V$ if $x \notin \varphi(X)$ and if $x \in \varphi(X \cup\{y\})$, then $y \in \varphi(X \cup\{x\})$.

Theorem 3.2 Let $G$ be a group with the exchange property, i.e. $\forall x, y \in G$ and for every subset $X \subseteq G$,

if $x \notin\langle X\rangle \wedge x \in\langle X \cup\{y\}\rangle$, then $y \in\langle X \cup\{x\}\rangle$. Then the order of every element $a \in G, a \neq 1$ is a prime.

Proof. First, we prove that every cyclic subgroup of $G$ is simple, i.e. every cyclic subgroup does not contain non trivial normal subgroup.

Suppose that $\{1\} \leq\langle x\rangle \leq\langle y\rangle$ for $x, y \in G$. Then $x \notin\{1\}$ and $x \in$ $\langle\{1\} \cup\{y\}\rangle$ such that substituting $X=\{1\}$ in $(3-1)$ we find $y \in\langle\{1\} \cup\{x\}\rangle=$ $\langle x\rangle$ and we get a contradiction with our assumption. Thus $O(x) \in\{p, q\}, \forall x \in$ $G \backslash\{1\}$.

Theorem 3.3 Let $G$ be a $p$-group such that $p$ is a prime. Then $G$ is a group with the exchange property if and only if $G$ is elementary abelian $p$-group. 
Proof. Suppose that $G$ is a $p$-group with the exchange property. Then by theorem (3-2)

$$
x^{p}=1, \forall x \in G, \quad(3-2)
$$

hence $G^{p}=\{1\}$ and by [10] $\Phi(G)=G^{p} G^{\prime}$. Since $G$ is a $p$-group, then

$$
\Phi(G)=G^{\prime}, \quad \Phi^{2}(G)=G^{\prime \prime}, \ldots
$$

If $G^{\prime}=\{1\}$, then $G$ is an elementary abelian group.

Suppose that $G^{\prime} \neq\{1\}$. Then there exist elements $a, b, c \in G$ such that

$$
[a, b]=a^{-1} b^{-1} a b=c \neq 1 . \quad(3-3)
$$

Now assume that $c \in\langle a\rangle$, then $a \in\langle c\rangle$. Let consider the subgroup, which is generated by two elements $a, b$, i.e. $\langle a, b\rangle$. If $\langle a, b\rangle$ is a cyclic group, then it is commutative and we have a contradiction with $(3-3)$, then $a \notin\langle b\rangle$ and $b \notin\langle a\rangle$. Hence the set $\{a, b\}$ forms a basis of group $\langle a, b\rangle$. Since $\langle a\rangle=\langle c\rangle$, so $\langle a, b\rangle=\langle c, b\rangle$ and by the basis property of $G[6]$. Thus we have that the set $\{c, b\}$ forms a basis of $G$ and this is a contradiction with properties of the Frattini subgroup, i.e.c $\in \Phi(G)$.

Hence $c \notin\langle a\rangle$ and $c \in\langle a, b\rangle$, and by the exchange property we have $b \in\langle a, c\rangle$. But then $\langle a, b\rangle=\langle a, c\rangle$. So by the basis property for $G$ and since $a \notin\langle\mathrm{b}\rangle, b \notin\langle\mathrm{a}\rangle$ we conclude that the set $\{a, c\}$ forms a basis for $G$. Hence this is a contradiction with properties of the the Frattini subgroup $\Phi(G)$, i.e. $c \in \Phi(G)$. Thus $[a, b]=1$ and the group $G$ is an elementary abelian $p$-group.

Conversely, suppose that a group $G$ is an elementary abelian $p$-group, then we consider $G$ as an additive group of a vector space over the field $G F(p)$.

Hence the exchange property is satisfied for a group $G$.

\section{Intersection between the basis property and the ex- change property}

Example 4.1 Let $S$ be the semilattice $\{a, b, 0\}$, where $a, b$ are incomparable and $a b=0$. Then $\mathrm{S}$ has unique basis, so $S$ has basis property. But $0 \in\langle\langle a\rangle \cup\{b\}\rangle$ and $0 \notin\langle\langle a\rangle\rangle, b \notin\langle\langle a\rangle \cup\{0\}\rangle$. Hence $S$ does not satisfy the exchange property.

Example 4.2 Let $G=\langle a\rangle$ be a cyclic group such that $|G|=p^{2}, p$ is a prime. Then $G$ is a group with the basis property, because it is a $p$-group, but it does not satisfy the exchange property. 
Theorem 4.3 Let $G$ be a finite group. Then $G$ is a group with the exchange property if and only if one of the following conditions hold:

1. $G$ is an elementary abelian $p$-group, $p$ is a prime.

2. $G$ is a semidirect product of an elementary abelian $p$-group $P$ by a cyclic $q$-group $\langle y\rangle$, of order $q$, where $p \neq q$ ( $p$ and $q$ are primes).Therefore $G$ must satisfy the following relations:

$$
\begin{gathered}
p \equiv 1(\bmod q), y^{-1} a y=a^{r}, r \in \mathbb{Z}^{+}, \\
r \not \equiv 1(\bmod p), r^{q} \equiv 1(\bmod p) .
\end{gathered}
$$

Proof. Suppose that $G$ is a group with the exchange property. Then we consider two cases:

Firstly, if $G$ is a primary group ( $p$-group), $p$ is a prime, then by theorem(33) $G$ is an elementary abelian $p$-group for a prime $p$.

Secondly, if $G$ is not primary group, then from the basis property in theorem(2-6), we see that $G$ is a semidirect product (i.e. $G=P \rtimes\langle y\rangle$ ) of $p$-group $P$ by a cyclic $q$-group $\langle y\rangle$, where $p \neq q$ ( $p$ and $q$ are primes). Since $P$ is a group with the exchange property, then by theorem(3-3) $P$ is an elementary abelian $p$ - group. Therefore by theorm(3-1) the group $\langle y\rangle$ has order $q, q$ is a prime.

Suppose that $|P|=p^{s}, s \in \mathbb{Z}^{+}$. Since the element $y$ is regular operator on $P$, i.e. the operator $\varphi$ inducing by element $y$ is a regular, then

$$
p^{s} \equiv 1(\bmod q) .
$$

Assume that $a \in P, a \neq 1$. Consider the element $b=y^{-1} a y$, since the operator $\varphi$ induced by element $y$ is regular, then $b \neq a$. Assume that $b \in\langle a\rangle$, hence $b=a^{r}, r \not \equiv 1(\bmod p)$. From $y^{q}=1$ we have $a^{r^{q}}=1$, i.e. $r^{q} \equiv 1(\bmod p)$.

Now let $b \notin\langle a\rangle$, so by the exchange property if $b \in\langle y, a\rangle$, then $y \in$ $\langle a, b\rangle \leq P$. We get a contradiction with $y \notin P$. Thus the automorphism $\varphi_{y}: P \rightarrow P$ is regular and act on a group $\langle a\rangle$ of order $p$, hence $p \equiv 1(\bmod q)$ and $p>q$. Since $G$ is a group with the basis property, then by theorem(2-6) the representation $y \rightarrow \varphi_{y}$ is an isotopic with dimension 1, i.e. the matrix $A$ of linear operator $\varphi_{y}$ in some basis of vector space $P$ which contains $s$ elements has the following form:

$$
A=\left(\begin{array}{ccc}
\bar{r} & 0 \ldots & 0 \\
0 & \bar{r} \ldots & 0 \\
0 & 0 & \bar{r}
\end{array}\right),
$$


such that $\bar{r}$ is an image of the element $r$ under the conical homomorphism $\bar{\theta}: \mathbb{Z} \rightarrow \mathbb{Z} / p \mathbb{Z}$, then

$r \not \equiv 1(\bmod p)$, and

$$
r^{q} \equiv 1(\bmod p) .
$$

Conversely, If $G$ is an elementary abelian $p$-group for a prime $p$, then $G$ is a group with basis property. Using theorem(3-3), then it remains to prove that if $P$ is an elementary abelian, and $\langle y\rangle$ has order $q$, where $p \neq q(p$ and $q$ are primes), and if the following conditions hold

$$
\begin{gathered}
y^{-1} x y=x^{r}, \forall x \in P \\
p \equiv 1(\bmod q) \\
r \neq 1(\bmod p) \\
r^{q} \equiv 1(\bmod p) .
\end{gathered}
$$

Then $G$ is a group with the exchange property. Suppose that the set $X \subseteq G$ and $a, b \in G$ such that $a \notin\langle X\rangle$ and $a \in\langle X \cup\{b\}\rangle$. Now we prove that $b \in\langle X \cup\{a\}\rangle$.

Let $G_{1}=\langle X \cup\{b\}\rangle$ and we study the following cases:

If $\langle X \cup\{b\}\rangle \leq P$, then $G_{1} \leq P, G_{1}$ satisfies the exchange property, because it is an elementary abelian $p$-group (by theorem(3-3)), hence $b \in$ $\langle X \cup\{a\}\rangle$.

If $\langle X \cup\{b\}\rangle \not \leq P$, then suppose that the set $X \cup\{b\}$ contains element of order $q$. Now if $X$ contains elements of order $q$, and since $G$ is a semidirect product of $p$-group by cyclic $\langle y\rangle$. Then we can prove that the set $X$ contains only one element of order $q$, (because if there exist two elements as $y^{s_{1}} a_{1}$, $y^{s_{2}} a_{2}$ in $X$ of order $q$, then for some $w \in \mathbb{Z}$ there exists $c \in P$ such that

$$
y^{s_{2}} a_{2}=\left(y^{s_{1}} a_{1}\right)^{w} c .
$$

Then $\left\langle y^{s_{1}} a_{1}, \quad c\right\rangle=\left\langle y^{s_{1}} a_{1}, \quad y^{s_{2}} a_{2}\right\rangle$, hence we consider element $y^{s_{2}} a_{2}$ as $c \in$ $P)$. Now suppose that the set $X=\left\{x_{1}, x_{2}, \ldots, x_{n}\right\}$ such that $x_{2}, \ldots, x_{n} \in P$, $x_{1} \notin P$. Then the Fitting subgroup $F(\langle X\rangle)$ of group $\langle X\rangle$ is generated by the set $\left\{x_{2}, \ldots, x_{n}\right\}$ and the image of this set under the automorphism $\varphi_{x_{1}}^{m}$, $m \in \mathbb{Z}$.

Since the group $P$ is an abelian group, then the Fitting subgroup $F(\langle X\rangle)$ is generated by the set $\left\{x_{2}, \ldots, x_{n}\right\}$ and the image of this set under the automorphism $\varphi_{y}^{m}$ and by $(4-1)$ this is the power of the same elements $x_{2}, \ldots, x_{n}$. In another words, the group $F(\langle X\rangle)$ is generated by $x_{2}, \ldots, x_{n}$ if these elements are exists. So by our assumption $a \in\langle X \cup\{b\}\rangle$. Then there exists a word $u\left(x_{1}, x_{2}, \ldots, x_{n}\right)$ such that $a=u\left(x_{1}, x_{2}, \ldots, x_{n}, b\right)$ and by $(4-1)$ we have

$$
a=v\left(x_{1}, x_{2}, \ldots, x_{n}\right) b^{w}
$$


such that $v\left(x_{1}, x_{2}, \ldots, x_{n}\right)$ is a word. If $b^{w}=e$, then by $(4-2)$ we have

$$
a=v\left(x_{1}, x_{2}, \ldots, x_{n}\right) \in\langle X\rangle .
$$

Thus we get a contradiction with our assumption for $a$, so we assume that $b^{w} \neq e$. Since a group $P$ is an elementary abelian $p$-group, then $\left\langle b^{w}\right\rangle=\langle b\rangle$, so by (4-2) we have

$$
b \in\left\langle b^{w}\right\rangle=\left\langle v\left(x_{1}, x_{2}, \ldots, x_{n}\right)^{-1} a\right\rangle \subseteq\langle X \cup\{a\}\rangle .
$$

Finally, let $X \subseteq P$. Since $X \cup\{b\} \nsubseteq P$, then $b$ is element of order $q$. Suppose that $G_{1}=\langle X \cup\{b\}\rangle$ is a semidirect product of a group $\langle X\rangle$ by $\langle b\rangle$. Then from $a \in\langle X \cup\{b\}\rangle$ we have the following for $w \in \mathbb{Z}$ and $c \in\langle X\rangle$

$$
a=b^{w} c
$$

If an element $a$ is a $q$-element, then $b^{w} \neq e$ and since $\langle b\rangle=\left\langle b^{w}\right\rangle$ we get

$$
b \in\left\langle b^{w}\right\rangle=\left\langle c^{-1} a\right\rangle \subseteq\langle X \cup\{a\}\rangle .
$$

If $a$ is $p$-element, then by $(4-3)$ we have $b^{w}=e$ and $a=c \in\langle X\rangle$ which is a contradiction with $a \notin\langle X\rangle$. Thus we study all cases. Hence $G$ is a group with change property.

\section{Acknowledgements}

The authors gratefully acknowledge the partial financial support from the Deanship of Academic Research (Project No.331201 ) at Al Imam Mohammad Ibn Saud Islamic University (IMSIU), Riyadh, Saudi Arabia.

\section{References}

[1] A. Al Khalaf, Finite group with basis property, Dok. Acad. Nauk BSSR, n. 11, 1989 (Russian)

[2] M. Alkadhi, A. Al Khalaf and M. Quick, The Nilpotency Class of Fitting Subgroups of Groups with Basis Property. International Journal of Algebra, Vol. 6, 2012, no. 14, 697 - 704.

[3] A. Aljouiee, Basis Property Conditions on Some Groups, International Journal of Mathematics and Computer Science, 3, No3, 1-11, (2008), Lebanon. 
[4] Jonathan McDougall-Bagnall, Martyn Quick, Groups with the basis property,Journal of Algebra 346 (2011) 332-339.

[5] P. R. Jones, A basis theorem for free inverse Semigroup, J. Algebra, v. 49, 1977. p.172-190.

[6] P. R. Jones, Basis properties for inverse Semigroups, J. Algebra, v. 50, 1978. p.135-152.

[7] P. R. Jones, Basis properties, exchange properties and embeddings in idempotent-free semigroups, Semigroups and their applications, 69-82, Reidel, Dordrecht, 1987.

[8] P. R. Jones, Exchange properties and basis properties for closure operators, Colloq. Math. 57(1989), no. 1, 29-33.

[9] M. Hall, The Theory of Groups, Macmillan, 1959.

[10] B. Huppert, Endliche Gruppen, Springer-Verlag, 1967.

[11] D. Robinson, A Course in the Theory of Groups, Second Edition.SpringerVerlag New York, Inc. (1996)

Al Khalaf Ahmad,

Department of Mathematics, College of Sciences

Al-Imam Mohammad Ibn Saud Islamic University

P.O. Box 90950, Riyadh, Saudi Arabia.

Email: akhalaf59@gmail.com

Alkadhi Mohammed

Department of Mathematics, College of Sciences

Al-Imam Mohammad Ibn Saud Islamic University

P.O. Box 90950, Riyadh, Saudi Arabia.

Email: malki2@yahoo.com 